\title{
Linear and Non-Linear Response in T-Shaped Electron Waveguides
}

\author{
M. BEK ${ }^{a}$, B.R. BuŁKA ${ }^{a}$ AND J. WRÓBEL ${ }^{b}$ \\ ${ }^{a}$ Institute of Molecular Physics, Polish Academy of Sciences, M. Smoluchowskiego 17, 60-179 Poznań, Poland \\ ${ }^{b}$ Institute of Physics, Polish Academy of Sciences, al. Lotników 32/46, 02-668 Warszawa, Poland \\ We present theoretical studies of three-terminal ballistic junction in linear and non-linear regime. Various \\ conductance and voltage dips and peaks are observed and their origin is explained as influence of the bend \\ resistance and the threshold effect.
}

PACS numbers: 73.23.-b, 73.63.Rt, 85.35.Ds, 85.35.Be

\section{Introduction}

Recent experimental results on three-terminal ballistic junction (TBJ) [1] showed their interesting electron transport properties as candidates for effective logic gates for quantum computing [2]. A similar device was proposed by Baranger [3] where T-shaped ballistic junction and four-terminal devices were examined. It was shown that oscillatory behavior of transmission coefficients can be explained accurately by bend resistance and threshold effect. In addition for specific parameters he observed appearance of the Wigner cusp [4] in transmission coefficients. In this paper we will show that the Wigner singularities can be observed in measurable quantities, such as conductance and voltage.

\section{Modelling of three-terminal ballistic devices}

The model of the TBJ consists of three semi-infinite strips of atoms and the coupling region (see Fig. 1). Calculations have been performed using tight-binding approach [5]. In a linear and non-linear regime respectively, we assumed that the voltage biased to the electrodes does not change the Fermi energy in the device. Using the Landauer-Büttiker approach we derive the current flowing through the $i$-th electrode (in linear response regime) $I_{i}=(2 e / h) \sum_{j} T_{i j}\left(\mu_{i}-\mu_{j}\right)$, where $T_{i j}$ is the transmission coefficient between $i$-th and $j$-th electrode, $\mu_{i, j}$ are the electrochemical potentials in the electrodes. The conductance measured between $i$-th and $j$ th electrode when the $k$-th electrode is left unbiased, is $\mathcal{G}_{i j, k}=\left(2 e^{2} / h\right)\left[T_{i j}+\left(T_{i k} T_{j k}\right) /\left(T_{i k}+T_{j k}\right)\right]$. The voltage on the unbiased electrode can be calculated using following form $V_{k}=\left(T_{i k} V_{i}+T_{j k} V_{j}\right) /\left(T_{i k}+T_{j k}\right)$. In our consideration we assume low temperature limit. That choice was made because at high temperature all the calculated quantities (i.e. conductance or voltage) will be blurred. Calculations and relations between transmission coefficient and the Green function are well established [6].

\section{The linear regime}

Let us consider the straight configuration (Fig. 1a) for the linear regime. For strong coupling between electrodes (Fig. 1c) electrons can easily penetrate the floating electrode. With decrease of the Fermi energy $E_{\mathrm{F}}$ transmission coefficients $T_{21}$ and $T_{32}$ increase but we observe dips near every threshold Fermi energy due to opening new transmission channel. The calculated conductance $\mathcal{G}_{12,3}$ is quantized but its plateaux are suppressed below multiple of $2 e^{2} / h$. Small dips in conductance plot near every threshold Fermi energy are the result of back actions in the floating electrode. Voltage on the floating electrode remains constant because in this case $T_{31}=T_{32}$. For the situation when a small additional potential $\epsilon_{2}=0.25$ is applied to the drain electrode (Fig. 1d) we observe in the transmission coefficients threshold singularities near every threshold Fermi energy. Calculated conductance shows sharp peaks and dips (as a result of appearing of the Wigner singularities in transmission plots) with small plateaux. Voltage on the floating electrode manifests oscillatory behavior because of difference between transmission coefficients between the floating electrode and the source/drain electrodes. We state that the Wigner singularities can be observed experimentally for low temperature limit in the conductance/voltage versus Fermi energy characteristics.

The bend configuration is presented in Fig. 1b. For strong coupling of the electrodes (Fig. 1e) conductance exhibits small plateaux, but still it is suppressed. Large dips in the conductance plot are the result of bend resistance. Voltage $V_{2}$ shows a sharp curvature and its maximum is connected with strong injection of electrons to the floating electrode. An additional local potential $\epsilon_{3}=0.25$ applied to the drain electrode (Fig. 1f) destroys the conductance quantization. Voltage calculated on the floating electrode $V_{2}$ shows peaks near threshold energies. Electrons escaping from the floating electrode cause increase of the voltage $V_{2}$. 
a)
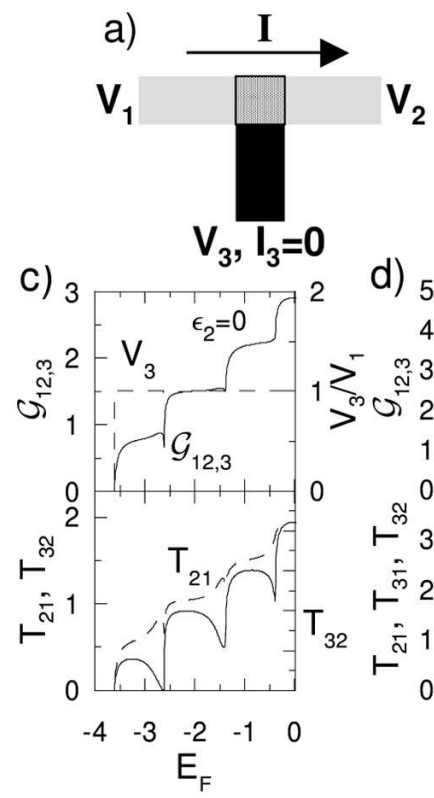

d) 5

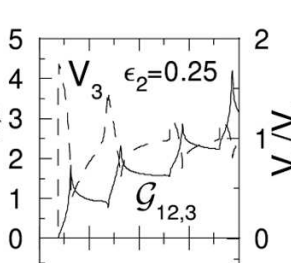

b)

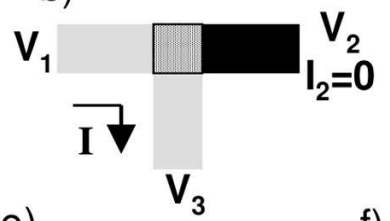

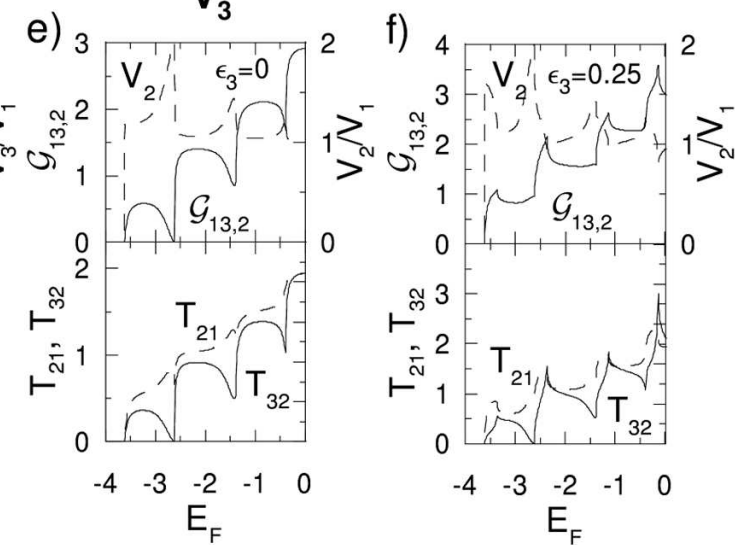

Fig. 1. (a) The straight configuration where source and drain voltage is applied to the electrodes 1 and 2 , (b) the bend configuration with source and drain voltage is applied to electrodes 1 and 3. For the linear regime the drain electrode is grounded as $V_{2}=0$ for the straight and $V_{3}=0$ for the bend configuration, respectively. For the non-linear regime $V_{1}=V / 2$ and $V_{2}=-V / 2$ for the straight and $V_{3}=-V / 2$ for the bend configuration, respectively. For all cases source and drain electrodes are marked as grey one, floating electrode (detector electrode) is marked as black. Dotted area in the center of device is the coupling region. Conductance $\mathcal{G}_{12,3}$ (solid curve) and voltage $V_{3}$ (dashed curve) versus $E_{\mathrm{F}}$ for the straight configuration: (c) $\epsilon_{2}=0, \epsilon_{3}=0$ and (d) $\epsilon_{2}=0.25, \epsilon_{3}=0$. Part (e) and (f) show $\mathcal{G}_{13,2}$ and $V_{2}$ for $\epsilon_{2}=0, \epsilon_{3}=0$ and $\epsilon_{2}=0, \epsilon_{3}=0.25$, respectively. In the bottom transmission coefficients $T_{21}$ (dashed curve), $T_{31}$ (dotted curve) and $T_{32}$ (solid curve) are shown. For all cases $t_{01}=t_{02}=t_{03}=-1, \epsilon_{1}=0, V_{1}=0.1$ and $M_{1}=M_{3}=4$.

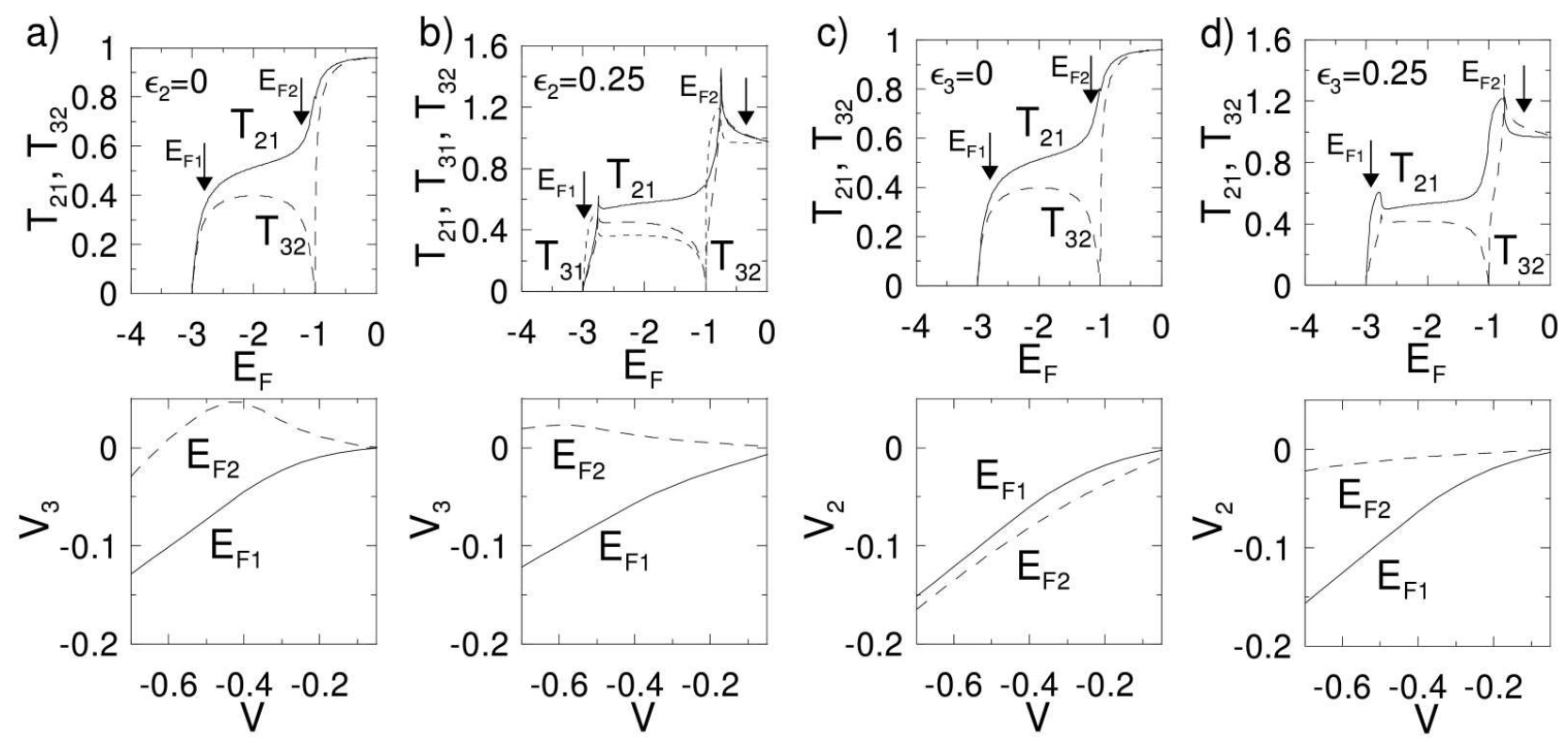

Fig. 2. Transmission coefficients $T_{21}$ (solid curve), $T_{31}$ (dotted curve) and $T_{32}$ (dashed curve) versus Fermi energy (top) and voltage on the floating electrodes $i, V_{i}$ versus applied bias voltage $V$ (bottom) for the straight configuration in (a) $\epsilon_{2}=0, \epsilon_{3}=0$ and (b) $\epsilon_{2}=0.25, \epsilon_{3}=0$; for the bend configuration in (c) $\epsilon_{2}=0, \epsilon_{3}=0$ and (d) $\epsilon_{2}=0, \epsilon_{3}=0.25$. Arrows indicate the Fermi energies $E_{\mathrm{F} 1}$ (solid curve) and $E_{\mathrm{F} 2}$ (dashed curve) for which the voltage on the detector electrode was calculated. For all cases $t_{01}=t_{02}=t_{03}=-1, \epsilon_{1}=0, V_{1}=0.1$ and $M_{1}=M_{3}=2$. 


\section{Non-linear regime}

In the non-linear regime the current in the floating electrode $i$ is $I_{i}=(2 e / h) \sum_{j \neq i} \int \mathrm{d} E T_{i j}(E)\left[f\left(E-\mu_{i}\right)\right.$ $\left.-f\left(E-\mu_{j}\right)\right]=0$, where $f$ are the Fermi distribution functions.

The results for the straight configuration are presented in Fig. 2a,b for strong coupling of the electrodes. The first value we choose $E_{\mathrm{F} 1}$ lies in the conduction band close to the transmission step $T_{i j}$. The voltage $V_{3}$ is always negative because the slope of transmission $T_{i j}$ is positive. Second value $E_{\mathrm{F} 2}$ lies in the bend resistance region, where the slope of the $T_{32}$ is negative. For $V>0.4$ we observe changing the slope of $V_{3}-V$ curve due to contributions from the second conduction channel. Applying small local potential $\epsilon_{2}=0.25$ (see Fig. 2b) to the drain electrode changes the transmission coefficients slightly (the Wigner cusps appear), but still one can observe negative values of $V_{3}$ for $E_{\mathrm{F} 1}$ and positive values of $V_{3}$ for wide range of applied bias voltage for $E_{\mathrm{F} 2}$.

The results for bend configuration are presented in Fig. 2c,d. For this configuration transmission coefficient $T_{21}$ and $T_{32}$ are merging together and in a result voltage on the detector electrode $V_{2}$ is always negative.

Behavior of TBJ's in non-linear limit is consistent with explanation of $\mathrm{Xu}$ [7]. The positive/negative values of voltage on the floating electrode depends on slope of the transmission curve. The negative/positive slope of the transmission curve yields positive/negative values of voltage on the floating electrode.

\section{Summary}

Summarizing we show that threshold effect can be seen in characteristics of source-drain conductance and voltage in the floating electrode. With this paper we encourage to perform such an experiment to observe these phenomena.

\section{Acknowledgments}

The work was support, as part of ESF EUROCORES Programme FoNE, by funds from the Ministry of Science and Higher Education for 2006-2009.

\section{References}

[1] A. Ramamoorthy, J.P. Bird, J.L. Reno, J. Phys., Condens. Matter 19, 276205 (2007); J. Wróbel, private communication.

[2] D. Csontos, H.Q. Xu, Phys. Rev. B 67, 235322 (2003); P.R. Bandaru, C. Daraio, S. Jin, A.M. Rao, Nature Mater. 4, 663 (2005).

[3] H.U. Baranger, Phys. Rev. B 42, 11479 (1990).

[4] E.P. Wigner, Phys. Rev. 73, 1002 (1948).

[5] E.N. Economou, Green's Function in Quantum Physics, 3rd ed., Springer, Berlin 2006.

[6] B.R. Bułka, A. Tagliacozzo, Phys. Rev. B 79, 075436 (2009).

[7] H.Q. Xu, Appl. Phys. Lett. 78, 2064 (2001). 